\title{
Erratum to: Humanistic Education and students' Educational Motivation in Tehran Primary Schools
}

\author{
PariNaz PourAli $^{1} \cdot$ Maryam SeifNaraghi $^{1}$ • \\ Ezatollah Naderi ${ }^{1}$
}

(C) Springer Science+Business Media New York 2016

\section{Erratum to: International Journal of Mental Health and Addiction 10.1007/s11469-016-9703-1}

Please note that the following is the correct full affiliation for the authors of this article:

Department of Educational Sciences, Science and Research Branch, Islamic Azad University, Tehran, Iran. Iran 